\title{
Spontaneous, Short-term Interaction with Mobile Robots
}

\author{
J. Schulte \\ C. Rosenberg \\ School of Computer Science \\ Carnegie Mellon University \\ Pittsburgh, PA 15213
}

S. Thrun

\begin{abstract}
Human-robot interaction has been identified as one of the major open research directions in mobile robotics. This paper considers a specific type of interaction: short-term and spontaneous interaction with crowds of people. Such patterns of interactions are found frequently when service robots operate in public places (e.g. information kiosks, receptionists, tourguide robots). This paper describes three components of a successfully implemented interactive robot: a motorized face as focal point for interaction, an architecture that suggests the robot has moods, and a method for learning how to interact with people. The approach has been implemented and tested using a mobile robot, which was recently deployed at a Smithsonian museum in Washington, DC. During a two week installation period it interacted with 50,000 people and we found that the robot's interactive capabilities were essential for its high on-task performance, and thus its practical success.
\end{abstract}

\section{Introduction}

Human-robot interaction has been identified as one of the major open research directions in mobile robotics.[4] Human-robot interaction is essential for an upcoming generation of service robots, which will have to directly interact with people. For example, service robots might assist elderly or handicapped people, assist humans in search-and-rescue missions, or perform janitorial services in environments populated by humans. Thus, interfaces for human-robot interaction are essential for the practical success of such systems. In all of the systems just described, the human-robot interaction is typically one-on-one and it is possible to train the user (and the robot) in the vocabulary of the interface. However, in certain service robot applications, such as robotic receptionists, information kiosks, or tour-guides, it is necessary for the robot to interact spontaneously, with completely untrained people who may not know the specific "vocabulary" of the interface.

In this article we focus on spontaneous short-term interaction. This is a type of human-robot interaction that will be typical for robotic applications such as information kiosks, receptionists, or tour-guides. These robots are often approached by groups of uninformed people, and typical interactions last for 10 minutes or less. This is in contrast to human-robot interfaces proposed by various researchers which utilize gesture, speech, clapping, and natural language based interfaces. These interfaces are generally effective for a specific class of interactions. Gestures, for example, are well-suited for directing a mobile robot to manipulate (e.g. pickup) specific objects.[8, 11, 16] Speech input has been demonstrated to be highly effective for tasks such as tele-operating robots, or attaching names to places in unknown environments.[1] However, such interfaces are targeted toward scenarios where a single person interacts with a robot. They typically fail when whole crowds of people interact with a robot.

In this work we focus on a tour-guide robot application. Tour-guide robots are usually approached by crowds of people, most of whom have never interacted with a robot before, and do not necessarily intend to do so when visiting a museum. In our system, a tourguide robot has three main goals during its operation:

- Traveling from one exhibit to the next during the course of a tour.

- Attracting people to participate in a new tour between tours.

- Engaging people's interest and maintaining their attention while describing a specific exhibit.

The main functional components necessary for the robot to accomplish these goals during its operation are navigation and interaction. By navigation, we mean the ability of the robot to localize itself in a 
map, plan a motion path to a target, and avoid obstacles. In many robotic systems navigation and its related subcomponents alone might be sufficient for the robot to accomplish its goals. However, in the application we are examining, the robot is in an environment crowded with people and one of its main functions is to provide a service to the people in its environment. To this end, interaction is as essential a component as navigation.

For the interaction to be effective, our approach is to create a system which acts in a believable manner while interacting with people in the context of spontaneous short-term interaction. A believable agent creates the impression that it is self-determining, and is an idea that has been considered in both software [2] and robotic [5] agents. We have created (and tested) a user interface for a robot with the goal of allowing it to act as a reasonable social agent in the specific context of the application described here, not under all possible conditions. In our approach, the three cornerstones which work together to create the impression of a believable agent are:

- Focal Point

- Emotional State

- Adaptation

The focal point provides people with a single location on which to focus their attention during interaction. In our implementation the focal point for human interaction was realized with a specific hardware interface consisting of a motorized face with pan and tilt control on top of the robot. The system communicates an emotional state to the people around it as a means of conveying its intention in a way that is easily understood in the context of a believable social agent. For example, a robot tour guide might have the intention of making progress while giving a tour. In our system the expression displayed on the motorized face and the contents of the recorded speech playback communicate this information. The adaptation is the ability of the system to learn from its interactions with people and modify its behavior to illicit the desired result.

We recently designed and installed such a robot, called Minerva, in the entrance area of the Smithsonian National Museum of American History, where it interacted with more than 50,000 people over a 2 -week period. In this paper we describe its basic architecture, and survey the results obtained in the museum. Our tour-guide robot Minerva had two basic intents: (1) to attract people to whom it could give a tour, and (2) to make progress while giving a tour. Both intents are somewhat orthogonal: while for the former, the robot wants people to come closer to it and

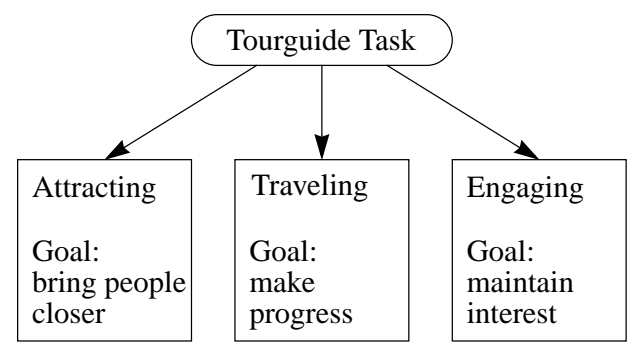

Figure 1: Decomposition of the tour guide interaction problem.

motivate their interest, the latter requires people to clear the way, hence stay behind the robot. This paper describes a number of mechanisms that were found essential in the pursuit of these two goals. It first describes a specific hardware interface, consisting or a motorized face, a pointable head and a voice synthesizer, which served as the focal point of human-robot interaction. It then describes two quite complimentary solutions, one for each goal described above. To make progress, the robot uses a simple stochastic finite state automaton, which communicates an emotional state or "mood" to the viewers. To attract people, Minerva uses a learning algorithm that enables it to adaptively determine the best action out of a pool of possible actions (speech acts, head motion primitives, and facial expressions).

To evaluate the utility of the proposed methods for spontaneous short-term interaction, this paper compares the Minerva robot with a different robot, called Rhino [3], which was built by the same group of researchers. In mid-1997, Rhino was installed as a robotic tour-guide in the Deutsches Museum Bonn. Both robots essentially use the same control and navigation software; the major difference lies in the nature of the interaction: Rhino did not possess any of the human-robot interfaces described in this paper. For example, instead of actively attracting people, Rhino just waited passively until people pushed a button, indicating their interest in a tour. Rhino used a very simple mechanism to communicate its intent to make progress when giving tours. As a result, Rhino's ability to attract people was much inferior when compared to Minerva, and it was much less effective when giving tours, as reflected by the rate of progress when moving from exhibit to exhibit. We largely attribute these differences to the interface, which proved essential for the Minerva's success and effectiveness. 


\section{Approach: Minerva The Robot}

We approach the problem of making Minerva a believable agent that uses interaction to reach its goals in three ways. First, a face is used to define a focal point for interaction. Second, the robot is supplied with an "emotional" state, expressed outwardly by facial expressions and sounds. Third, adaptation occurs in one of the interaction tasks using a memory based learner. We describe these aspects of Minerva, with an explanation of how each contributes to the goals of different tasks.

\subsection{The Face}

At this point in time, there exists little precedence for robotic interaction with novice users upon which to build a new system. Hence, to engage museum visitors, it was in our interest to present as recognizable and intuitive an interface as possible: a caricature of a human face. $[9,10,15]$ It was important that the face contain only those elements necessary for the degree of expression appropriate for a tour guide robot. A fixed mask would render the robot incapable of visually representing mood, while a highly accurate simulation of a human face would contain numerous distracting details beyond our control. An iconographic face consisting of two eyes with eyebrows and a mouth is almost universally recognizable, and can portray the range of simple emotions useful for tour guide interaction. Figure 2 shows three possible expressions realized by different configurations of the face hardware.

We determined, also, that a physically implemented face would be more convincing and interesting than a flat display.[9] Reasons for this include the expectation that moving objects require intelligent control, while flat moving images likely result from the playback of a stored sequence as in film or television. Additionally, a three-dimensional face can be viewed from many angles, allowing museum visitors to see it without standing directly in front of the robot.

The face has four degrees of freedom which were implemented via servo motors controlled by a serial port interface. One degree of freedom was used to separately control each eyebrows and two degrees of freedom were used to control the mouth. The choice of the number of degrees of freedom was made as much for ease of implementation as to facilitate display of the desired emotions. The face control motors are mounted on and arranged around a central box. The "eyes" of the robot were a pair of Sony XC-999 color cameras. These cameras were not used for navigation or obstacle avoidance in the system, but were present for the sole purpose of transmitting a robot's eye view of the museum to web visitors. The eyebrows, consisting of blue rectangles, are mounted directly above the cameras. The eyebrows can independently move \pm 90 degrees from horizontal. The mouth consisted of a red elastic band. Each end of the band was mounted to a servo control arm and its motion was constrained by three pins. Even though both sides of the mouth could be controlled independently, they were controlled in a coordinated fashion such to bring the "mouth" into a smiling or frowning configuration. Because of the arrangement of the degrees of freedom of the mouth and the bandwidth of the actuators, it was not possible to make the "lips" move in synchronization to the speech generated by the robot. Instead, a bar graph style LED display was mounted behind the mouth. The bars of the display illuminated in response to the speech generated by the robot. Two such displays were mounted in mirror image fashion back to back such that when the robot spoke, the length of the displayed bar increased symmetrically from the center of the mouth.

The head assembly was mounted on a Directed Perception PTU-46-17.5 pan tilt head. This allowed the head to be rotated approximately \pm 90 degrees from the centerline of the robot and allowed the head to tilt slightly from the horizontal.

The face hardware installed on Minerva served a second purpose beyond communicating its intent; it provided a focal point for the interaction between the human and the robot. By focal point, we refer to a place for a human to focus attention and better understand that the system will follow some basic social conventions. It aids the human interacting with the robot to anthropomorphize it.[9] People focused attention on Minerva's face when interacting with it. As anecdotal evidence, individuals tended to take photographs of just Minerva's face, whereas in the case of Rhino, people tended to take pictures of the entire robot. People understood that the robot's face pointed in the direction it intended to go, even when the robot was stopped. Similarly, the LEDs placed behind the mouth provided a focal point when speech was generated, which localized the sound there, even though the speech was actually produced at the robot base.

\subsection{Emotional State}

Minerva's emotional state is the basis of travelrelated interaction. Travel occurs between stops in a tour, when Minerva moves through the museum and finds its way to the next exhibit to discuss. To navi- 
(a)

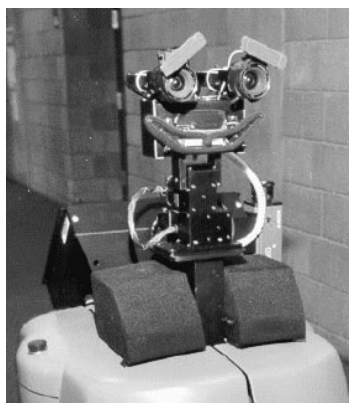

(b)

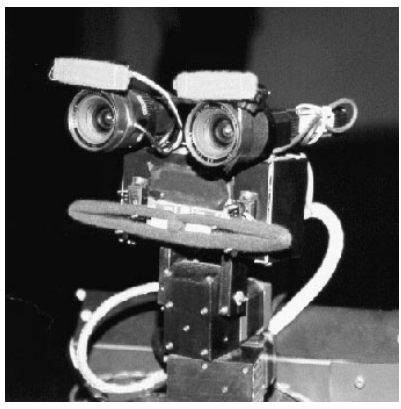

(c)

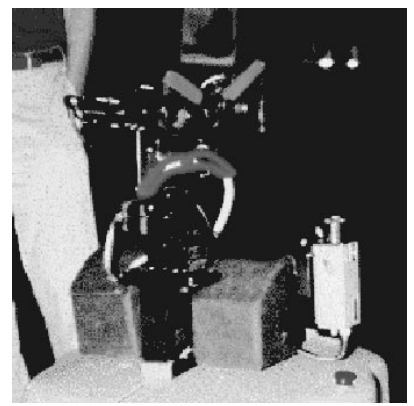

Figure 2: Minerva with (a) happy, (b) neutral, and (c) angry facial expressions.

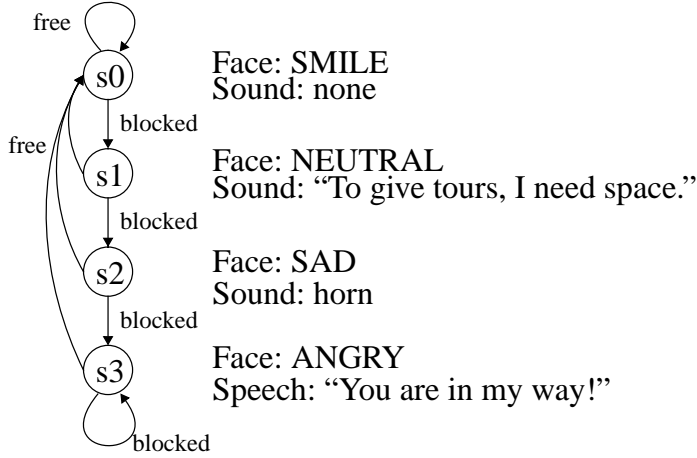

Figure 3: State diagram of Minerva's emotions during travel. "Free" and "blocked" indicate whether a person stands in the robot's path.

gate through crowded spaces, the robot must be able to decide whether an obstacle is a static object or is a human. This determination is achieved solely by the use of an entropy filter applied to the laser range data and the museum map.[7] If the robot is being blocked by a person, it needs to communicate its intent to those who are in the way. Possibly, the most effective way to do this would be to loudly and aggressively state that everyone should step away. However, another implicit objective of our robot is to interact in a friendly and socially acceptable manner. To communicate its intent to make progress in a particular direction, Minerva utilizes its interface: an expressive face, a pan/tilt head, and speech output. It is with these "effectors" that Minerva must manipulate the environment around it. Our solution is to combine these behaviors in a simple state machine, where the state is represented externally as a mood. Please note that by mood, we do not presume to suggest that this system has the property of "emotion," we simply use the term to indicate an emotional state that the person observing Minerva would impart to it.[5, 13] In this work we view "mood" from an engineering view point - it is nothing more than a means to an end. We feel this sets apart Minerva from other agents which utilize emotion as part of their interface $[6,12,14]$.

The emotional state machine is designed as follows. Minerva starts in a "happy" state, smiling while traveling between tour stops, until first confronted by a human obstacle that cannot be trivially bypassed. At this point, the robot kindly points out that it is giving a tour and changes to a neutral expression, while pointing its head in the direction it needs to travel. If this does not bring success, Minerva adopts a sad expression, and may ask the obstructing person to stand behind it. This usually makes sense in context, since the direction the head points suggests a "front" and "back" of the robot. If the person still does not move, then Minerva frowns and becomes even more demanding. A total of four states encode the complete travel interaction behavior, as shown in Figure 3 .

Emotional state helps Minerva achieve navigational goals by enhancing the robot's believability. Observation of interaction with museum visitors suggests that people are generally unconcerned about blocking the path of a passive, mute robot. A change of facial expression and sudden utterance by Minerva usually results in a quick response from anyone in the way. (One side-effect is that some people wish to find out how much they can perturb the robot, and will intentionally prevent it from moving.) Our subjective interpretation of the effect of emotional state is that the increasing "frustration" of the robot produces feelings of empathy in many people and coerces them to move. This empathy is possible, we think, because the timely and exaggerated transition of moods lends Minerva a believable personality in this limited context. 


\begin{tabular}{l|c} 
Feature & Values \\
\hline $\begin{array}{l}\text { facial expression } \\
\text { face pointing target } \\
\text { sound output }\end{array}$ & $\begin{array}{c}\text { happy, neutral, sad, angry } \\
\text { closest person, center of mass of people, least populated area, random direction }\end{array}$
\end{tabular}

Table 1: An action is performed by setting each of the three features to one of the pre-defined values listed above.

\subsection{Adaptation}

Between tours, Minerva spends approximately one minute generating interaction behaviors with the goal of attracting people to follow it on the next tour. We chose to experiment with learning interactive behaviors by having the robot select actions, then evaluate them based on the movement of people in the period of time following the new action. An action was defined to be a joint setting of three features: a facial expression, a pan/tilt target for pointing the face, and a sound type. A memory-based learner (MBL) was used to store the results of interaction experiences in order to make future decisions when confronted with the same task. A performance function mapped the sequence of movements by people following an action into a single scalar value that we refer to as a reward, indicating the relative success of the behavior. The function was defined such that an increase in closeness and density of people around the robot was rewarded and a decrease was penalized.

Interaction with humans by a robot presents a unique and challenging learning problem. The realm of possible actions with different meaning in an interaction setting is enormous. Subtle changes in the speech timing and volume, or in the intensity of a facial expression can affect the quality of interaction significantly. The effect of a given action is not constant, and much of the state that could help define specific state/action pairs is hidden to a robot with limited sensing capability. In particular, our robot is unable to detect anything more about the humans with whom it is interacting than their distances and spatial densities. However, a robot with a caricature face brings about the expectation of somewhat minimal interaction. One would not expect to carry on a complex discourse with a cartoon-like machine, though one may still find it interesting and worth approaching, despite its inability to emulate human behavior with any precision. Given this, we chose a very biased and limited, but learnable space of overall interaction possibilities. The range of possible robot behaviors was selected to include obviously "good" and "bad" actions, but the overall cadence of interaction was fixed.

Specifically, Minerva enters an "attraction interac- tion" state for one minute between museum tours, where the goal is to attract people in preparation for the next tour. In this state, an action is initiated consisting of facial expression, face pointing direction, and sound output. This action persists for 10 seconds, after which a new action is selected. During this interval, the distances and densities of people around the robot are monitored and used to evaluate the effect of the action. The evaluation result, or reward, is stored by the MBL. The next action is selected by choosing that which maximizes the expected reward given the learner's previous experiences and the current state. Some features of this new action are occasionally randomized to ensure that new regions of the action space are explored. The action space is outlined in Table 1.

After some experimentation we chose a very simple learning strategy. The MBL chooses an action $a$ such that:

$$
\max _{a \in A} m(a)
$$

where $A$ is the set of all 80 possible actions, and $m(a)$ is simply the mean of previous rewards following action a. If no experiences with a have been recorded, then $m(a)$ returns zero, which corresponds to the reward following an action that produces no change, positive or negative, in the distribution of people around the robot. The simplicity of this approach reflects the difficulty of collecting sufficient data in a noisy environment. The algorithm described above is on-line in the sense that learning occurs continuously and the results of experiments immediately affect future actions, without human intervention or the execution of a separate training step.

\section{$3 \quad$ Results}

\subsection{Travel Interaction}

In the museum environment a tour guide robot is often surrounded by people which impede its forward progress (Figure 4). An examination of the average speed of Minerva $(38.8 \mathrm{~cm} / \mathrm{s})$ showed it to navigate more quickly than the Rhino robot $(33.8 \mathrm{~cm} / \mathrm{s})$, even though Minerva operated in a considerably more populated environment. We attribute this to the fact that 
(a)

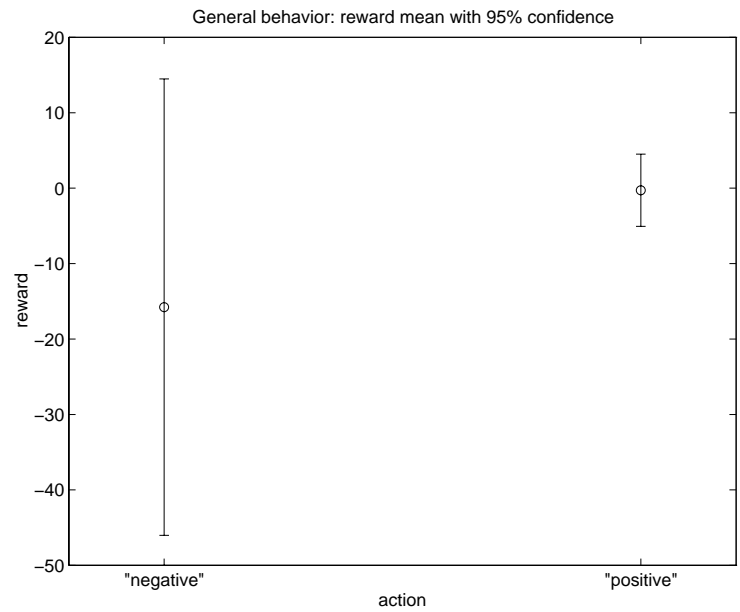

(b)

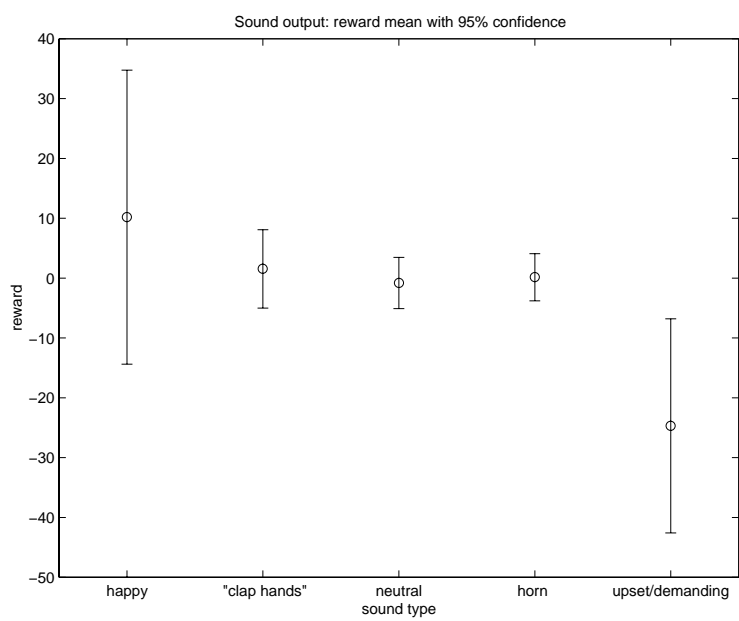

Figure 5: Minerva's expected reward for different types of actions are plotted above. (a) A comparison of "positive" (friendly) and "negative" (unfriendly) actions, and (b) five different categories of sounds produced by Minerva, with reward averaged over all other action features.

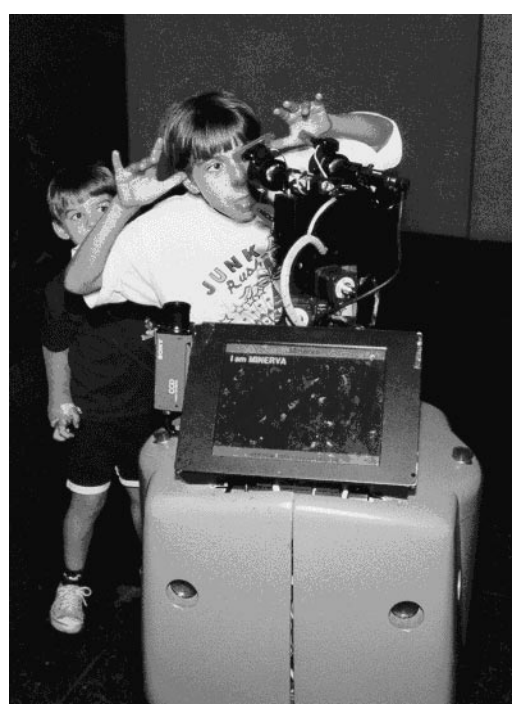

Figure 4: Interaction helps Minerva navigate through crowded environments.
Minerva could more efficiently and clearly indicate its intended direction of travel. Also, in terms of entertainment value, Minerva's behavior during this time is more interesting to the people who follow the robot. Others have also found interfaces similar to Minerva's to have entertainment value.[12, 14]

From observation, it was clear that museum visitors understood the changes in mood brought about by obstructing Minerva. While not everyone chose to move, the robot's expectations were quite clear. In the case of the faceless robot Rhino, a horn sound was used to clear people from its path obstructed. People found this signal to be ambiguous, and did little to impart the believability that helped Minerva influence people.

\subsection{Attraction Interaction}

Minerva performed 201 attraction interaction experiments, and over time become a more friendly robot that attracted people more successfully. A measure of distances of people from the robot is an inherently noisy measure of the success of an interaction behavior. Nevertheless, we have seen promising indications that some basic adaptation and parameter tuning within a pre-defined behavior can work to make an agent more flexible.

Ultimately, we expect that this flexibility can enhance believability. Figure 5 shows the learned expected reward for different types of behavior at the end of the experiments. The first plot compares "negative" and "positive" actions. Negative actions are 

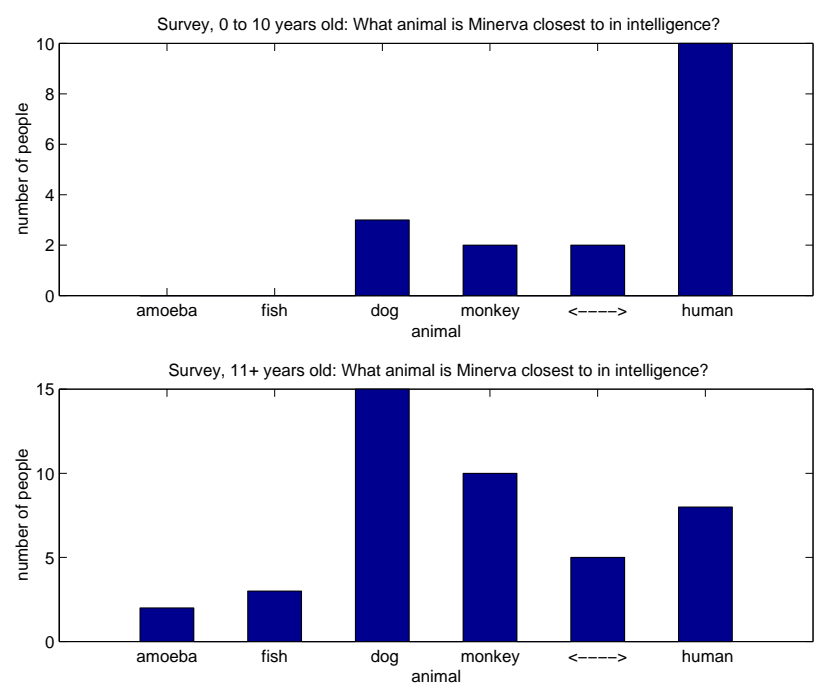

Figure 6: Histogram of survey responses comparing Minerva's intelligence to that of 5 animals for respondents (top) 0-10 years old, and (bottom) 11+ years old.

those for which Minerva makes a demand of the visitors in a stern voice while frowning. Positive actions consist of friendlier comments and a neutral or happy facial expression. The numbers were produced by taking a weighted average of the value of the expected reward function $m(a)$ for all actions belonging to the category being analyzed.

The second plot (Figure 5b) compares the expected reward resulting from the five categories of sound that Minerva can produce. Here we can see a clear tendency for happy sounds to produce greater reward than neutral sounds, and for upset sounds to result in a penalty. The fact that the horn sound falls in the neutral reward category sheds some light on the difficulty that Rhino had convincing people to move in previous research. While these figures are of limited significance, there is a promising trend of increasing reward with friendlier behavior. The larger confidence interval for "negative" actions reflects the fact that less data was collected by Minerva in this less promising region of the action space, since the exploration strategy was biased toward successful actions. Due to the noisiness of the data relative to the number of experiments, and the fact that we could perform only one training session, a plot of the performance increase over time would not be meaningful.

\subsection{Visitor Surveys}

To measure the subjective concept of Minerva's believability, we asked a sampling of 60 museum visitors to answer a short questionnaire. Perhaps the most interesting estimate of believability results from answers to the question: "As far as intelligence is concerned, what would be the most similar animal? (amoeba, fish, dog, monkey, or human)" Figure 6 shows histograms of the responses for the age group 0 to 10 years, and greater than 10 years. The bar between "monkey" and "human" is a count of respondents that suggested that Minerva fell somewhere between the two categories. Clearly, young children were more likely to attribute human-like intelligence to the robot. Most of this group (64felt that Minerva was "alive,", while very few others would make this assertion. For the questions that we asked, gender played little role in perception of Minerva. The notion of intelligence does not directly correspond to believability, but it is encouraging to find Minerva frequently compared to animals that we recognize as complex social creatures. For the questions that we asked, gender played little role in perception of Minerva.

\section{Summary and Conclusions}

Interfaces for human-robot interaction are essential for an upcoming generation of service robots, which will have to directly interact with people. In this paper we focus on interfaces targeted toward spontaneous, short-term interaction. The Minerva tour guide robot described in this paper is an example of a robot which interacts with people in this way.

Our experiments have demonstrated the usefulness of our approach for building such an interface. In our system this included: an expressive face, a head with pan and tilt control, and speech output. These systems allowed Minerva to be perceived as a believable agent and effectively communicate its intent to the individuals interacting with it. The Minerva robot was able to make progress through the museum during tours at the same rate as the Rhino robot, even though the Minerva robot encountered an order of magnitude more people. Both robots were similar, with the exception of the interaction component.

We experimented with both a hand coded solution and a learning based solution to action selection for this interface and found both to be effective. Because the space of possible interaction behaviors is so large, learning necessarily occurs within a limited action space. Nevertheless, we found that Minerva suc- 
cessfully learned to select actions that improved the effectiveness of interaction, using an on-line algorithm.

In conclusion, we have demonstrated that a robot system, with an interface that represents the robot as a believable social agent, can effectively exploit traditional social interactions between humans, to communicate intent during spontaneous, short-term interaction. We view this mode of interaction as another tool in the interface designer's tool box when building systems which need to interact with uninformed robot users and in environments where uninformed users may impede the robot in achieving its goals.

\section{Acknowledgments}

We would like to thank The Lemelson Center of the National Museum of American History for providing us a venue for conducting this research. And Greg Armstrong for the all-important task of keeping the hardware running.

We would also like to gratefully acknowledge our sponsors: DARPA via AFMSC (contract number F04701-97-C-0022), TACOM (contract number DAAE07-98-C-L032), and Rome Labs (contract number F30602-98-2-0137). Additional financial support was received from Daimler Benz Research and Andy Rubin.

\section{References}

[1] H. Asoh, S. Hayamizu, H. Isao, Y. Motomura, S. Akaho, and T. Matsui. Socially embedded learning of office-conversant robot Jijo-2. In Proceedings of IJCA I-97, pages 880-885, 1997.

[2] J. Bates. The role of emotion in believable agents. Technical Report CMU-CS-94-136, School of Computer Science, Carnegie Mellon University, Pittsburgh, PA, April 1994.

[3] W. Burgard, A. Cremers, D. Fox, D. Häehnel, G. Lakemeyer, D. Schulz, W. Steiner, and S. Thrun. The interactive museum tour-guide robot. In Proceedings of $A A A I-98$, pages 11-18, 1998.

[4] J. Crisman and G. Bekey. Grand challenges for robotics and automation: The 1996 ICRA panel discussion. ICRA-96, 1996.

[5] K. Dautenhahn. The role of interactive conceptions of intelligence and life in cognitive technology. In Proceedings of CT-97, pages 33-43, 1997.
[6] C. Breazeal (Ferrell). A motivational system for regulating human-robot interaction. In Proceedings of $A A A I-98$, pages 54-61, Madison, WI, 1998

[7] D. Fox, W. Burgard, S. Thrun, and A. Cremers. Position estimation for mobile robots in dynamic environments. In Proceedings of $A A A I-98$, pages 983-988, 1998 .

[8] R. Kahn, M. Swain, P. Prokopowicz, and R. Firby. Gesture recognition using the Perseus architecture. In Proceedings of the IEEE Conference on Computer Vision and Pattern Recognition, pages 734-741, 1996.

[9] W. King and J. Ohya. The representation of agents: Anthropomorphism, agency, and intelligence. In Proceedings of CHI-96, 1996.

[10] T. Koda. Agents with faces: The effect of personification. In 5th IEEE International Workshop on Robot and Human Communication, Tsukuba, Japan, November 1996.

[11] D. Kortenkamp, E. Huber, and P. Bonasso. Recognizing and interpreting gestures on a mobile robot. In Proceedings of AAAI-96, pages 915921, 1996.

[12] P. Maes. Artificial life meets entertainment: Interacting with lifelike autonomous agents. Special Issue on New Horizons of Commercial and Industrial AI, Communications of the ACM, 38(11):108-114, November 1995.

[13] S. Penny. Embodied cultural agents: At the intersection of art, robotics and cognitive science. In AAAI Socially Intelligent Agents Symposium, Cambridge, MA, November 1997.

[14] C. Rosenberg and C. Angle. IT: An interactive animatronic prototype. IS Robotics Inc. internal development project, description available at: http://www. cs. cmu. edu/ chuck/robotpg/itpg/, December 1994.

[15] A. Takeuchi and T. Naito. Situated facial displays: Towards social interaction. In Proceedings of CHI-95, 1995.

[16] S. Waldherr, S. Thrun, R. Romero, and D. Margaritis. Template-based recognition of pose and motion gestures on a mobile robot. In Proceedings of $A A A I-98,1998$. 